\title{
OUTER MEASURES AND WEAK REGULARITY OF MEASURES
}

\author{
DALE SIEGEL
}

Kingsborough Community College

Mathematics/Computer Science Department 2001 Oriental Boulevard Brooklyn. New York 11235

(Received January 29, 1992 and in revised form July 7, 1994)

ABSTRAC'T. This paper investigates smoothness properties of probability measures on lattices which imply regularity, and then considers weaker versions of regularity; in particular, weakly regular. vaguely regular. and slightly regular. They are derived from commonly used outer measures, and we analyze them mainly for the case of $I(\mathcal{L})$ or for those elements of $I(\mathcal{L})$ with added smoothness conditions.

KEY WORDS AND PHRASES. Lattice regular, $\sigma$-smooth, and outer measures. Weakly, vaguely, and slightly regular measures. Normal and complement generated lattices.

\section{AMS SUBJECT CLASSIFICATION CODE 28C15}

\section{INTRODUCTION}

Let $X$ be an arbitrary set and $\mathcal{L}$ a lattice of subsets of $X . A(\mathcal{C})$ denotes the algebra generated by $\mathcal{L}$ and $I(\mathcal{L})$ those non-trivial zero-one valued finitely additive measures on $A(\mathcal{L}) . I_{\sigma}(\mathcal{L})$ denotes those elements of $I(\mathcal{L})$ that are $\sigma$-smooth on $\mathcal{L}$; while $I_{R}(\mathcal{L})$ denotes those elements of $I(\mathcal{L})$ that are $\mathcal{L}$-regular. To each $\mu \in I(\mathcal{L})$ we will associate a finitely subadditive outer measure $\mu^{\prime}$ on $P(X)$, and to $\mu \in I_{\sigma}(\mathcal{L})$ is associated an outer measure $\mu^{\prime \prime}$. The relationships between $\mu^{\prime}$ and $\mu^{\prime \prime}$ on $\mathcal{L}$ and $\mathcal{L}^{\prime}$ (the complementary lattice) are investigated. We show, e.g., $\mu^{\prime}=\mu^{\prime \prime}$ on $\mathcal{L}^{\prime}$ if and only if $\mu \in J(\mathcal{L})$ : those $\mu \in I(\mathcal{L})$ such that for $L_{n} \downarrow \mathrm{L}, L_{n}, L \in \mathcal{L}, \mu(L)=\inf _{n} \mu\left(L_{n}\right)$. Conditions for $\mu^{\prime}=\mu^{\prime \prime}$ or $\mu=\mu^{\prime \prime}$ on $\mathcal{L}$ are also investigated. This leads to a consideration of weak notions of regularity, two of which can be expressed in terms of $\mu^{\prime}$ and $\mu^{\prime \prime}$. In this respect the normal lattices are particularly important since for such lattices regularity of $\mu$ coincides with weak regularity. We also show that if $\mu \in J(\mathcal{L})$ and if $\mathcal{L}$ is complement generated then $\mu$ is weakly regular. Combining these results gives conditions for certain measures to be regular. We also give a complete characterization of those $\mu \in I(\mathcal{L})$ which are slightly regular (see below for definitions). We adhere to standard lattice and measure terminology which will be used throughout the paper (see e.g. $[1,4,5]$ ) and review some of this in section two for the reader's convenience. 


\section{DEFINITIONS AND NOTATIONS}

Let $X$ be an abstract ret. Let $\mathcal{L}$ be a lattice of subsets of $X$. We assume throughout that $\emptyset$ and $X$ are in $\mathcal{L}$. If $A \subset A$, then we will denote the complement of $A$ by $A^{\prime}$ (i.e. $A^{\prime}=X^{\prime}-A$ ). If $\mathcal{L}$ is a lattice of subsets of $X$, then $\mathcal{L}^{\prime}=\left\{L^{\prime} \mid L \in \mathcal{L}\right\}$ is the complementany lattice of $\mathcal{L}$.

\section{Lattice Terminology}

(2.1) DEFINITION: Let $\mathcal{L}$ be a lattice of subsets of $X$. We say that:

$1-\mathcal{L}$ is a $\delta$-lattice if it is closed under countable intersections; $\delta(\mathcal{L})$ is the lattice of countable intersections of sets of $\mathcal{L}$.

2- $\mathcal{L}$ is complement generated if $L \in \mathcal{L}$ implies $L=\bigcap_{n=1}^{\infty} L_{n}^{\prime}$, where $L_{n} \in \mathcal{L}$.

3- $\mathcal{L}$ is countably paracompact if, for every sequence $\left\{L_{n}\right\}$ in $\mathcal{L}$ such that $L_{n} \downarrow \emptyset$, there exists a sequence $\left\{\tilde{L}_{n}\right\}$ in $\mathcal{L}$ such that $L_{n} \subset \tilde{L}_{n}^{\prime}$ and $\tilde{L}_{n}^{\prime} \downarrow \emptyset$.

4- $\mathcal{L}$ is disjunctive if and only if $x \in X, L \in \mathcal{L}$, and $x \notin L$ imply there exists $A, B \in \mathcal{L}$ such that $x \in A, L \subset B$, and $A \cap B=\emptyset$.

5- $\mathcal{L}$ is compact if and only if $X=\bigcup_{\alpha} L_{\alpha}{ }^{\prime}, L_{\alpha} \in \mathcal{L}$, implies there exists a finite number of $L_{\alpha}^{\prime}$ that cover $X$.

6- $\mathcal{L}$ is countably compact if and only if $X=\bigcup_{\imath=1}^{\infty} L_{\imath}^{\prime}, \quad L_{\imath} \in \mathcal{L}$, implies there exists a finite number of the $L_{2}^{\prime}$ that cover $X$.

7- $\mathcal{L}$ is normal if and only if $A, B \in \mathcal{L}$ and $A \cap B=\emptyset$ imply there exists $C, D \in \mathcal{L}$ such that $A \subset C^{\prime}, B \subset D^{\prime}$, and $C^{\prime} \cap D^{\prime}=\emptyset$.

\section{MEASURE TERMINOLOGY}

Let $\mathcal{L}$ be a lattice of subsets of $X . M(\mathcal{L})$ will denote the set of finite-valued, bounded, finitely additive measures on $A(\mathcal{L})$. We may clearly assume throughout that all measures are non-negative.

\section{(2.2) DEFINITIONS:}

1- A measure $\mu \in M(\mathcal{L})$ is said to be $\sigma$-smooth on $\mathcal{L}$ if $L_{n} \in \mathcal{L}$ and $L_{n} \downarrow \emptyset$ imply $\mu\left(L_{n}\right) \rightarrow 0$.

2- A measure $\mu \in M(\mathcal{L})$ is said to be $\sigma$-smooth on $A(\mathcal{L})$ if $A_{n} \in A(\mathcal{L})$ and $A_{n} \downarrow \emptyset$ imply $\mu\left(A_{n}\right) \rightarrow 0$.

3- A measure $\mu \in M(\mathcal{L})$ is said to be $\mathcal{L}$-regular if, for any $A \in A(\mathcal{L})$, $\mu(A)=\sup \{\mu(L): L \subset A, L \in \mathcal{L}\}$.

(2.3) NOTATIONS: If $\mathcal{L}$ is a lattice of subsets of $X$, then we will denote by:

$M_{\sigma}(\mathcal{L})=$ the set of $\sigma$-smooth measures on $\mathcal{L}$ of $M(\mathcal{L})$

$M^{\sigma}(\mathcal{L})=$ the set of $\sigma$-smooth measures on $A(\mathcal{L})$ of $M(\mathcal{L})$

$M_{R}(\mathcal{L})=$ the set of $\mathcal{L}$-regular measures of $M(\mathcal{L})$

$M_{R}^{\sigma}(\mathcal{L})=$ the set of $\mathcal{L}$-regular measures of $M^{\sigma}(\mathcal{L})$

\section{(2.4) DEFINITIONS:}

1- If $A \in A(\mathcal{L})$, then $\mu_{x}(A)=\{1$ if $x \in A$, and 0 if $x \notin A\}$ is the measure concentrated at 
$x \in X^{\circ}$.

2- $I(\mathcal{L})$ is the subset of $M(\mathcal{L})$ which consists of non-trivial zero-one measures.

The respective zero-one valued subsets of (2.3) are designated by

$I_{\sigma}(\mathcal{L}), I^{\sigma}(\mathcal{L}), I_{R}(\mathcal{L})$ and $I_{R}^{\sigma}(\mathcal{L})$.

\section{(2.5) DEFINITIONS:}

1- Let $\mu \in M(\mathcal{L})$. Then $\mu \in N(\mathcal{L})$ if $L_{n} \in \mathcal{L}$ and $\bigcap_{n=1}^{\infty} L_{n}=L \in \mathcal{L}$ (in particular, if $\mathcal{L}$ is $\delta$ ). $L_{n} \downarrow$, imply $\mu(L)=\inf \mu\left(L_{n}\right)$.

2- Let $\mu \in I(\mathcal{L})$. Then $\mu \in J(\mathcal{L})$ if $L_{n} \in \mathcal{L}$ and $\bigcap_{n=1}^{\infty} L_{n}=L \in \mathcal{L}, L_{n} \downarrow$, imply $\mu(L)=\inf \mu\left(L_{n}\right)$.

(2.6) DEFINITIONS:

1- If $\mu \in M(\mathcal{L})$, then the support of $\mu$ is $S(\mu)=\cap\{L \in \mathcal{L} \mid \mu(L)=\mu(X)\}$.

2- If $\mu \in I(\mathcal{L})$, then $S(\mu)=\cap\{L \in \mathcal{L} \mid \mu(L)=1\}$.

\section{(2.7) REMARKS:}

1 - $I(\mathcal{L})$ is in one-to-one correspondence with the set of all prime $\mathcal{L}$ - filters.

2- $I_{\sigma}(\mathcal{L})$ is in one-to-one correspondence with prime $\mathcal{L}$ - filters which have the countable intersection property.

3- $I_{R}(\mathcal{L})$ is in one-to-one correspondence with the set of all $\mathcal{L}$-ultrafilters.

\section{SEPARATION TERMINOLOGY}

(2.8) DEFINITIONS: Let $\mathcal{L}_{1}$ and $\mathcal{L}_{2}$ be two lattices of subsets of X.

1- $\mathcal{L}_{1}$ semi-separates $\mathcal{L}_{2}$ if $A_{1} \in \mathcal{L}_{1}, A_{2} \in \mathcal{L}_{2}$, and $A_{1} \cap A_{2}=\emptyset$ imply there exists $B_{1} \in \mathcal{L}_{1}$ such that $A_{2} \subset B_{1}$ and $A_{1} \cap B_{1}=\emptyset$.

2- $\mathcal{L}_{1}$ separates $\mathcal{L}_{2}$ if $A_{2}, B_{2} \in \mathcal{L}_{2}$ and $A_{2} \cap B_{2}=\emptyset$ imply there exists $A_{1}, B_{1} \in \mathcal{L}_{1}$ such that $A_{2} \subset A_{1}, B_{2} \subset B_{1}$, and $A_{1} \cap B_{1}=\emptyset$.

3- Let $\mathcal{L}_{1} \subset \mathcal{L}_{2} . \mathcal{L}_{2}$ is $\mathcal{L}_{1}$-countably bounded if, for any sequence $\left\{B_{n}\right\}$ of sets of $\mathcal{L}_{2}$ with $B_{n} \downarrow \emptyset$, there exists a sequence $\left\{A_{n}\right\}$ of sets of $\mathcal{L}_{1}$ such that $B_{n} \subset A_{n}$ and $A_{n} \downarrow \emptyset$.

(2.9) REMARKS: Listed below are a few basic important facts that will be used throughout the paper (see $[2,3,6]$ for further details and related matters).

1- If $\mu \in M(\mathcal{L})$, then there exists $\nu \in M_{R}(\mathcal{L})$ such that $\mu \leq \nu(\mathcal{L})$ (i.e. $\mu(L) \leq \nu(L)$, all $L \in \mathcal{L})$ and $\mu(X)=\nu(X)$.

2- $\mathcal{L}$ is disjunctive if and only if $\mu_{x} \in I_{R}(\mathcal{L})$, all $x \in X$.

3- $\mathcal{L}$ is countably compact if and only if $I(\mathcal{L})=I_{\sigma}(\mathcal{L})$.

4- Suppose $\mu \leq \nu(\mathcal{L})$, where $\mu \in M(\mathcal{L})$ and $\nu \in M_{R}(\mathcal{L})$. If $\mathcal{L}$ is normal , then $\nu\left(L^{\prime}\right)=\sup \left\{\mu(\tilde{L}): \tilde{L} \subset L^{\prime} ; \quad L, \tilde{L} \in \mathcal{L}\right\}$.

5- Suppose $\mu \in M_{R}(\mathcal{L})$ and $\gamma \in M_{R}\left(\mathcal{L}^{\prime}\right)$ such that $\mu \leq \gamma\left(\mathcal{L}^{\prime}\right)$. Then $\mathcal{L}$ is normal if and only if $\mu\left(L^{\prime}\right)=\sup \left\{\gamma(A): A \subset L^{\prime} ; A, L \in \mathcal{L}\right\}$.

6- Suppose $\mathcal{L}$ is normal and complement generated. Then $\mu \in N(\mathcal{L})$ implies $\mu \in M_{R}^{\sigma}(\mathcal{L})$.

7- Suppose $\mathcal{L}_{1} \subset \mathcal{L}_{2}$. Extend $\mu_{1} \in I\left(\mathcal{L}_{1}\right)$ to $\mu_{2} \in I\left(\mathcal{L}_{2}\right)$ and extend $\nu_{1} \in I_{R}\left(\mathcal{L}_{1}\right)$ to 
$\nu_{2} \in I_{R}\left(\mathcal{L}_{2}\right)$. If $\mathcal{L}_{1}$ s'parates $\mathcal{L}_{2}$ and $\mu_{1} \leq \nu_{1}\left(\mathcal{L}_{1}\right)$, then $\mu_{2} \leq \nu_{2}\left(\mathcal{L}_{2}\right)$.

8- Suppose $\mathcal{L}_{1} \subset \mathcal{L}_{2}$. If $\mathcal{L}_{1}$ separates $\mathcal{L}_{2}$, then $\mathcal{L}_{1}$ is normal if and only if $\mathcal{L}_{2}$ is normal.

\section{OUTER MEASURES}

In this section we consider $\mu \in M(\mathcal{L})$, and associate with it certain "outer measures" $\mu^{\prime}$ and $\mu^{\prime \prime}$. In general, they differ from the customary induced "outer

measures" $\mu^{\bullet}$ and $\mu^{*}$. We seck to investigate the interplay of these outer measures on the lattice $\mathcal{L}$ and, conversely, the effect of $\mathcal{L}$ on them. We will consider mainly the case where $\mu \in I(\mathcal{L})$, and for this reason we will usually restrict discussion of $\mu^{\prime \prime}$ to the case where $\mu \in I_{\sigma}(\mathcal{L})$ since, otherwise, $\mu^{\prime \prime} \equiv 0$.

(3.1) DEFINITIONS: Let $\mu \in M(\mathcal{L})$ such that $\mu \geq 0$ and let $E$ be a subset of $X$.

1- $\mu^{\prime}(E)=\inf \left\{\mu\left(L^{\prime}\right): E \subset L^{\prime}, L \in \mathcal{L}\right\}$ is a finitely-subadditive outer measure.

2- $\mu^{\prime \prime}(E)=\inf \left\{\sum_{n=1}^{\infty} \mu\left(L_{n}^{\prime}\right): E \subset \bigcup_{n=1}^{\infty} L_{n}^{\prime}, L_{n} \in \mathcal{L}\right\}$ is a countably-subadditive outer measure.

3- $\mu^{\bullet}(E)=\inf \{\mu(A): E \subset A, A \in A(\mathcal{L})\}$ is a finitely-subadditive outer measure.

4- $\mu^{*}(E)=\inf \left\{\sum_{\imath=1}^{\infty} \mu\left(A_{\imath}\right): E \subset \bigcup_{\imath=1}^{\infty} A_{\imath}, A_{\imath} \in A(\mathcal{L})\right\}$ is a countably-subadditive outer measure.

(3.2) DEFINITION: $\nu$ is said to be a regular outer measure (or regular finitelysubadditive outer measure) if $\nu$ is an outer measure (finitely subadditive) and if, for $A, E \subset X$, there exists $E \in \mathcal{S}_{\nu}$ (where $\mathcal{S}_{\nu}$ denotes the $\nu$-measurable sets) such that $A \subset E$ and $\nu(A)=\nu(E)$.

\section{(3.3) PROPERTIES:}

1- Suppose $\mathcal{L}$ is $\delta$ and let $\mu \in N(\mathcal{L})$ (or just $\mu \in J(\mathcal{L})$ ).

Then $\mu\left(\bigcup_{i=1}^{\infty} L_{i}^{\prime}\right) \leq \sum_{i=1}^{\infty} \mu\left(L_{i}^{\prime}\right), L_{i} \in \mathcal{L}, i=1,2, \ldots$

2- Let $\mu \in M_{\sigma}(\mathcal{L})$. Then: $(a) \mu^{\prime \prime}(X)=\mu(X),(b) \mu \leq \mu^{\prime \prime} \leq \mu^{\prime}(\mathcal{L}),(c) \mu^{\prime \prime} \leq \mu=\mu^{\prime}\left(\mathcal{L}^{\prime}\right)$.

3- Let $\mu \in I_{\sigma}(\mathcal{L})$. If $\mu\left(L_{k}\right)=1$, all $k, L_{k} \in \mathcal{L}$, then $\mu^{\prime \prime}\left(\bigcap_{k=1}^{\infty} L_{k}\right)=1$.

4- Suppose $\nu$ is a finitely-subadditive, regular outer measure defined on $P(X)$, the set of all subsets of $X$. Then $E \in \mathcal{S}_{\nu}$ if and only if $\nu(X)=\nu(E)+\nu\left(E^{\prime}\right)$.

5- If $\mu \in J(\mathcal{L})$ and $\mathcal{L}$ is $\delta$, then $\mu^{\prime \prime}=\mu^{\prime}(\mathcal{L})$.

PROOF: We will just prove 2 and 5.

2. (a) Clearly $\mu^{\prime \prime}(X) \leq \mu(X)$. If $\mu^{\prime \prime}(X)<\mu(X)$, then there exists $L_{\mathfrak{\imath}}^{\prime} \in \mathcal{L}^{\prime}$, $i=1,2, \ldots$, such that $X=\bigcup_{\imath=1}^{\infty} L_{\imath}^{\prime}$ and $\sum_{\imath=1}^{\infty} \mu\left(L_{\imath}^{\prime}\right)<\mu(X)$.

But $\sum_{\imath=1}^{\infty} \mu\left(L_{\imath}^{\prime}\right)=\lim _{n \rightarrow \infty} \sum_{1}^{n} \mu\left(L_{\imath}^{\prime}\right) \geq \lim _{n \rightarrow \infty} \mu\left(\bigcup_{1}^{n} L_{\imath}^{\prime}\right)$. Also $\bigcup_{1}^{n} L_{\imath}^{\prime} \uparrow X$ and $\bigcup_{1}^{n} L_{\imath}^{\prime} \in \mathcal{L}^{\prime}$.

This implies that $\lim _{n} \mu\left(\bigcup_{1}^{n} L_{\imath}^{\prime}\right)=\mu(X)$ since $\mu \in M_{\sigma}(\mathcal{L})$. Therefore $\mu^{\prime \prime}(X)=\mu(X)$.

(b) Suppose there exists $L \in \mathcal{L}$ such that $\mu(L)>\mu^{\prime \prime}(L)$. Then

$$
\mu^{\prime \prime}(X) \leq \mu^{\prime \prime}(L)+\mu^{\prime \prime}\left(L^{\prime}\right)<\mu(L)+\mu^{\prime \prime}\left(L^{\prime}\right)
$$


But $\mu^{\prime \prime} \leq \mu(\mathcal{L})$. implyng $\mu^{\prime \prime}\left(X^{\prime}\right)<\mu(L)+\mu\left(L^{\prime}\right)=\mu\left(X^{-}\right)$. This contradicts $(a)$.

Hence $\mu \leq \mu^{\prime \prime}(\mathcal{L})$; and $\mu^{\prime \prime} \leq \mu^{\prime}$ everywhere clearly.

Thus $\mu^{\prime \prime} \leq \mu^{\prime}(\mathcal{L})$. Therefore $\mu \leq \mu^{\prime \prime} \leq \mu^{\prime}(\mathcal{L})$.

(c) Clearly $\mu^{\prime \prime} \leq \mu^{\prime}\left(\mathcal{L}^{\prime}\right)$ and, by definition, $\mu=\mu^{\prime}\left(\mathcal{L}^{\prime}\right)$. Therefore $\mu^{\prime \prime} \leq \mu=\mu^{\prime}\left(\mathcal{L}^{\prime}\right)$.

5. Suppose $\mathcal{L}$ is $\delta$ and $\mu \in J(\mathcal{L})$. Then, by (3.3.1), $\mu\left(\bigcup_{\imath=1}^{\infty} L_{\imath}^{\prime}\right) \leq \sum_{i=1}^{\infty} \mu\left(L_{\imath}^{\prime}\right)$, and $\mu \in$ $J(\mathcal{L}) \Longrightarrow \mu \in I_{\sigma}(\mathcal{L}) \Longrightarrow \mu \leq \mu^{\prime \prime} \leq \mu^{\prime}(\mathcal{L})$. Now suppose $\mu^{\prime \prime}(L)=0$ and $\mu^{\prime}(L)=1, L \in \mathcal{L}$. Then $L \subset \bigcup_{n=1}^{\infty} L_{n}^{\prime}, \quad L_{n} \in \mathcal{L}(n=1,2, \ldots)$, and $\mu\left(L_{n}^{\prime}\right)=0$, all $n$. This implies $\mu\left(L_{n}\right)=1$. all $n$, and $L \subset\left(\bigcap_{n=1}^{\infty} L_{n}\right)^{\prime}=\tilde{L}^{\prime} \in \mathcal{L}$ since $\mathcal{L}$ is $\delta$. Hence, since $\mu \in J(\mathcal{L}), \mu(\tilde{L})=\inf _{n} \mu\left(L_{n}\right)$. Consequently $\mu(\tilde{L})=1$, which implies $\mu\left(\tilde{L}^{\prime}\right)=0$. Thus $\mu^{\prime}(L)=0$, a contradiction. Therefore $\mu^{\prime \prime}=\mu^{\prime}(\mathcal{L})$.

(3.4) THEOREM: If $\mu \in I(\mathcal{L})$, then

$$
\mathcal{S}_{\mu^{\prime}}=\left\{E \subset \mathcal{X}^{-} \mid E \supset L, \mu(L)=1, L \in \mathcal{L}, \text { or } E^{\prime} \supset L, \mu(L)=1, L \in \mathcal{L}\right\} .
$$

PROOF: Since $\mu \in I(\mathcal{L}), \mu^{\prime}$ is regular and therefore, by (3.3.4), to show $E \in \mathcal{S}_{\mu^{\prime}}$ it is enough to show that $\mu^{\prime}(X)=\mu^{\prime}(E)+\mu^{\prime}\left(E^{\prime}\right)$. The proof now follows directly.

(3.5) COROLLARY: If $\mu \in I(L)$, then $\mathcal{S}_{\mu^{\prime}} \cap \mathcal{L}=\left\{L \in \mathcal{L} \mid \mu(L)=\mu^{\prime}(L)\right\}$.

(3.6) THEOREM: If $\mu \in I_{\sigma}(\mathcal{L})$, then

$$
\begin{aligned}
\mathcal{S}_{\mu^{\prime \prime}}= & \left\{E \supset \bigcap_{n=1}^{\infty} L_{n}, \mu\left(L_{n}\right)=1, \text { all } n, L_{n} \in \mathcal{L},\right. \text { or } \\
& \left.E^{\prime} \supset \bigcap_{n=1}^{\infty} L_{n}, \mu\left(L_{n}\right)=1, \text { all } n, L_{n} \in \mathcal{L}\right\} .
\end{aligned}
$$

\section{PROOF:}

1.Again, $\mu \in I_{\sigma}(\mathcal{L})$ implies $\mu^{\prime \prime}$ is a non-trivial regular outer-measure. So $E \in \mathcal{S}_{\mu^{\prime \prime}}$ if and only if $\mu^{\prime \prime}(X)=\mu^{\prime \prime}(E)+\mu^{\prime \prime}\left(E^{\prime}\right)$, and the proof follows.

2. Suppose $E \supset \bigcap_{n=1}^{\infty} L_{n}, \mu\left(L_{n}\right)=1$, all $n, L_{n} \in \mathcal{L}$. Then $E^{\prime} \subset \cup L_{n}^{\prime}$ and $\mu\left(L_{n}^{\prime}\right)=0$, all $n$, which imply $\mu^{\prime \prime \prime}\left(E^{\prime}\right)=0$. Thus $\mu^{\prime \prime}(E)=1$. Therefore $\mu^{\prime \prime}(X)=\mu^{\prime \prime}(E)+\mu^{\prime \prime}\left(E^{\prime}\right)$, and, by (3.3.3), $E \in \mathcal{S}_{\mu^{\prime \prime}}$. Similarly $E^{\prime} \supset \bigcap_{n=1}^{\infty} L_{n}, \mu\left(L_{n}\right)=1$, all $n, L_{n} \in \mathcal{L}$ imply $E \in \mathcal{S}_{\mu^{\prime \prime}}$.

(3.7) THEOREM: If $\mu \in I_{\sigma}(\mathcal{L})$, then $\mathcal{S}_{\mu^{\prime \prime}} \cap \mathcal{L}=\left\{L \in \mathcal{L} \mid \mu(L)=\mu^{\prime \prime}(L)\right\}$ if and only if $\mu \in J(\mathcal{L})$.

\section{PROOF:}

1. Suppose $\mathcal{S}_{\mu^{\prime \prime}} \cap \mathcal{L}=\left\{L \in \mathcal{L} \mid \mu(L)=\mu^{\prime \prime}(L)\right\}$. Let $L_{n} \downarrow L, L_{n} \in \mathcal{L}, L \in \mathcal{L}$. Then $\bigcap_{n=1}^{\infty} L_{n}=$ $L$. Suppose $\mu\left(L_{n}\right)=1$, all $n$, but $\mu(L)=0$. Then $\mu^{\prime \prime}\left(L_{n}\right)=1$, all $n$, which implies $L_{n} \in \mathcal{S}_{\mu^{\prime \prime}} \cap \mathcal{L}$. It follows that $\cap L_{n}=L \in \mathcal{S}_{\mu^{\prime \prime}} \cap \mathcal{L}$, but $\mu^{\prime \prime}$ is countably additive on $\mathcal{S}_{\mu^{\prime \prime}}$. Hence $\mu^{\prime \prime}(L)=\lim _{n \rightarrow \infty} \mu^{\prime \prime}\left(L_{n}\right)=1$. Thus $\mu(L)=1$ since $L \in \mathcal{S}_{\mu^{\prime \prime}} \cap \mathcal{L}$, a contradiction. Therefore $\mu \in J(\mathcal{L})$.

2. Suppose $\mu \in J(\mathcal{L})$. Clearly $\mathcal{S}_{\mu^{\prime \prime}} \cap \mathcal{L} \supset\left\{L \in \mathcal{L} \mid \mu(L)=\mu^{\prime \prime}(L)\right\}$. 
Let $L \in\left\{L \in \mathcal{L} \mid \mu(L)=\mu^{\prime \prime}(L)\right\}$. Then

$$
\mu^{\prime \prime}\left(X^{\prime}\right)=\mu(L)+\mu\left(L^{\prime}\right) \geq \mu^{\prime \prime}(L)+\mu^{\prime \prime}\left(L^{\prime}\right)
$$

since $\mu(L)=\mu^{\prime \prime}(L)$ and $\mu^{\prime \prime} \leq \mu\left(\mathcal{L}^{\prime}\right)$. Hence $L \in \mathcal{S}_{\mu^{\prime \prime}} \cap \mathcal{L}$. Thus, by $(3.6), L \supset \bigcap_{n=1}^{\infty} L_{n}$. $\mu\left(L_{n}\right)=1$, all $n, L_{n} \in \mathcal{L}$, or $\bigcap_{1}^{\infty} L_{n} \subset L^{\prime}, \mu\left(L_{n}\right)=1, L_{n} \in \mathcal{L}$, all $n$. It can be shown that $L \in\left\{L \in \mathcal{L} \mid \mu(L)=\mu^{\prime \prime}(L)\right\}$. Thus $\mathcal{S}_{\mu^{\prime \prime}} \cap \mathcal{L} \subset\left\{L \in \mathcal{L} \mid \mu(L)=\mu^{\prime \prime}(L)\right\}$.

Therefore $\mathcal{S}_{\mu^{\prime \prime}} \cap \mathcal{L}=\left\{L \in \mathcal{L} \mid \mu(L)=\mu^{\prime \prime}(L)\right\}$.

(3.8) THEOREM: Suppose $\mu \in J(\mathcal{L}), \mathcal{L}$ is $\delta$, and $\mathcal{L} \subset \mathcal{S}_{\mu^{\prime \prime}}$. Then $\mu \in I_{R}^{\sigma}(\mathcal{L})$.

PROOF: Suppose $\mathcal{L} \subset \mathcal{S}_{\mu^{\prime \prime}}$. Then $\mathcal{L}=\mathcal{S}_{\mu^{\prime \prime}} \cap \mathcal{L}$. Hence, by (3.3.4), $\mathcal{L}=\mathcal{S}_{\mu^{\prime}} \cap \mathcal{L}$, which implies $\mu \in I_{R}(\mathcal{L})$. Clearly $\mu \in I_{\sigma}(\mathcal{L})$. Therefore $\mu \in I_{R}^{\sigma}(\mathcal{L})$.

(3.9) THEOREM: If $\mathcal{L}$ is countably compact and if $\mu \in I(\mathcal{L})$, then $\mu^{\prime \prime}=\mu^{\prime}(\mathcal{L})$.

PROOF: Suppose $\mathcal{L}$ is countably compact and $\mu \in I(\mathcal{L})$.

Then $\mu \in I_{\sigma}(\mathcal{L})$ by $(2.9 .3)$, which implies $\mu \leq \mu^{\prime \prime} \leq \mu^{\prime}(\mathcal{L})$ by (3.3.1). Now suppose there exists $L \in \mathcal{L}$ such that $\mu^{\prime \prime}(L)=0$ and $\mu^{\prime}(L)=1$. Then there exists $L_{n} \in \mathcal{L}, n=1,2, \ldots$, such that $L \subset \bigcup_{n=1}^{\infty} L_{n}^{\prime}$ and $\mu\left(L_{n}^{\prime}\right)=0$, all $n$. By the definition of countably compact, $L \subset \bigcup_{1}^{N} L_{\imath}^{\prime}=\tilde{L}^{\prime} \in \mathcal{L}^{\prime} ;$ and $\mu\left(\tilde{L}^{\prime}\right)=\mu\left(\bigcup_{1}^{N} L_{\imath}^{\prime}\right) \leq \sum_{1}^{N} \mu\left(L_{\imath}^{\prime}\right)=0$.

Hence $\mu^{\prime}(L)=0$, a contradiction. Therefore $\mu^{\prime}=\mu^{\prime \prime}(\mathcal{L})$.

(3.10) THEOREM: $\mu \in I_{\sigma}(\mathcal{L})$ and $\mu^{\prime \prime}=\mu^{\prime}\left(\mathcal{L}^{\prime}\right)$ if and only if $\mu \in J(\mathcal{L})$.

\section{PROOF:}

1. Suppose $\mu \in I_{\sigma}(\mathcal{L})$ and $\mu^{\prime}=\mu^{\prime \prime}\left(\mathcal{L}^{\prime}\right)$, but $\mu \notin J(\mathcal{L})$. Then there exists $L, L_{n} \in \mathcal{L}$ $(n=1,2, \ldots)$ such that $L_{n} \downarrow L, \mu\left(L_{n}\right)=1$ (all $\left.n\right)$, but $\mu(L)=0$. Thus $L^{\prime}=\bigcup_{n=1}^{\infty} L_{n}^{\prime}$ and $\mu\left(L^{\prime}\right)=1$, but $\sum_{n=1}^{\infty} \mu\left(L_{n}^{\prime}\right)=0$. Hence $\mu^{\prime \prime}\left(L^{\prime}\right)=0$, a contradiction since $\mu^{\prime \prime}=\mu^{\prime}=\mu\left(\mathcal{L}^{\prime}\right)$. Therefore $\mu \in J(\mathcal{L})$.

2. Suppose $\mu \in J(\mathcal{L})$. Then $\mu \in I_{\sigma}(\mathcal{L})$ and this implies $\mu^{\prime \prime} \leq \mu^{\prime}=\mu\left(\mathcal{L}^{\prime}\right)$. Now suppose, for some $L^{\prime} \in \mathcal{L}^{\prime}, \mu^{\prime \prime}\left(L^{\prime}\right)=0$ and $\mu^{\prime}\left(L^{\prime}\right)=1$. Then, by definition of $\mu^{\prime \prime}$, there exists $L_{n} \in \mathcal{L}, n=1,2, \ldots$, such that $L^{\prime} \subset \bigcup_{1}^{\infty} L_{n}^{\prime}$ and $\mu\left(L_{n}^{\prime}\right)=0$, all $n$. Thus $L^{\prime} \subset \bigcup_{1}^{\infty}\left(L_{n}^{\prime} \cap L^{\prime}\right)=\bigcup_{1}^{\infty} A_{n}^{\prime}$, where $A_{n}=L_{n} \cup L \in \mathcal{L}$ and $\mu\left(A_{n}^{\prime}\right)=0$, all $n$. Consequently $L=\cap A_{n}$, and $\mu(L)=0$ since $\mu\left(L^{\prime}\right)=\mu^{\prime}\left(L^{\prime}\right)=1$. Hence $\mu\left(A_{n}\right)=1$, all $n$, which is a contradiction since $\mu \in J(\mathcal{L})$. Therefore $\mu^{\prime}=\mu^{\prime \prime}\left(\mathcal{L}^{\prime}\right)$.

(3.11) THEOREM: Suppose $\mu \leq \nu(\mathcal{L})$, where $\mu \in M(\mathcal{L})$ and $\nu \in M_{R}(\mathcal{L})$. Then:

(a) $\mu \leq \nu=\nu^{\prime} \leq \mu^{\prime}(\mathcal{L})$

(b) if $\mathcal{L}$ is normal, then $\nu^{\prime}=\mu^{\prime}(\mathcal{L})$.

\section{PROOF:}

(a) Since $\nu \in M_{R}(\mathcal{L}), \nu(E)=\nu^{\prime}(E)=\inf \left\{\nu\left(L^{\prime}\right): E \subset L^{\prime}, L \in \mathcal{L}\right\}$. 
Alno, $\mu \leq \nu(\mathcal{L})$ implies $\nu \leq \mu\left(\mathcal{L}^{\prime}\right)$, which implies $\nu^{\prime} \leq \mu^{\prime}(\mathcal{L})$ and $\nu^{\prime} \leq \mu^{\prime}\left(\mathcal{L}^{\prime}\right)$.

Therefore $\mu \leq \|=1^{\prime} \leq \mu^{\prime}(\mathcal{L})$.

(b) Lot $L \in \mathcal{L}$. Then. by nonnality.

$$
\begin{aligned}
\nu^{\prime}(L) & =\nu(L)=\nu\left(\mathrm{X}^{-}\right)-\nu\left(L^{\prime}\right) \\
& =\nu\left(\mathrm{X}^{-}\right)-\sup \left\{\nu(\tilde{L}): \tilde{L} \subset L^{\prime}, \tilde{L} \in \mathcal{L}\right\} \\
& =\nu\left(X^{\circ}\right)-\sup \left\{\mu(\tilde{L}): \tilde{L} \subset L^{\prime}, \tilde{L} \in \mathcal{L}\right\} \\
& =\inf \left\{\mu\left(\tilde{L}^{\prime}\right): \tilde{L}^{\prime} \supset L\right\}=\mu^{\prime}(L) .
\end{aligned}
$$

\section{WEAKER NOTIONS OF REGULARITY}

Previously we have considered some properties related to $\mu \in M_{R}(\mathcal{L})$ or $\mu \in I_{R}(\mathcal{L})$. We now want to consider weaker notions of regularity, and see when they might coincide with regularity; and, in general, to investigate their properties and interplay with the underlying lattice.

(4.1) DEFINITIONS: Let $L \in \mathcal{L}$, where $\mathcal{L}$ is a lattice of subsets of $X$.

1- A measure $\mu \in M(\mathcal{L})$ is said to be weakly regular if

$$
\mu\left(L^{\prime}\right)=\sup \left\{\mu^{\prime}(\tilde{L}): \tilde{L} \subset L^{\prime}, \tilde{L} \in \mathcal{L}\right\} .
$$

2- A measure $\mu \in M_{\sigma}(\mathcal{L})$ is said to be vaguely regular if

$$
\mu\left(L^{\prime}\right)=\sup \left\{\mu^{\prime \prime}(\tilde{L}): \tilde{L} \subset L^{\prime}, \tilde{L} \in \mathcal{L}\right\} .
$$

3- A measure $\mu \in I(\mathcal{L})$ is thus weakly regular if $\mu\left(L^{\prime}\right)=1$ implies $L^{\prime} \supset \tilde{L} \in \mathcal{L}$ such that $\mu^{\prime}(\tilde{L})=1$.

4- A measure $\mu \in I_{\sigma}(\mathcal{L})$ is thus vaguely regular if $\mu\left(L^{\prime}\right)=1$ implies $L^{\prime} \supset \tilde{L} \in \mathcal{L}$ such that $\mu^{\prime \prime}(\tilde{L})=1$.

5- A measure $\mu \in I_{\sigma}(\mathcal{L})$ is said to be slightly regular if $\mu\left(L^{\prime}\right)=1$ implies $L^{\prime} \supset \bigcap_{n=1}^{\infty} L_{n}$ such that $\mu\left(L_{n}\right)=1, L_{n} \in \mathcal{L}$, all $n$.

\section{(4.2) NOTATIONS:}

$M_{W}(\mathcal{L})=$ the set of weakly regular measures of $M(\mathcal{L})$

$M_{V}(\mathcal{L})=$ the set of vaguely regular measures of $M_{\sigma}(\mathcal{L})$

$I_{W}(\mathcal{L})=$ the set of weakly regular measures of $I(\mathcal{L})$

$I_{V}(\mathcal{L})=$ the set of vaguely regular measures of $I_{\sigma}(\mathcal{L})$

$I_{S}(\mathcal{L})=$ the set of slightly regular measures of $I_{\sigma}(\mathcal{L})$

(4.3) LEMMA: $I_{R}^{\sigma}(\mathcal{L}) \subset I_{V}(\mathcal{L}) \subset I_{W}(\mathcal{L}) \cap I_{\sigma}(\mathcal{L})$

(4.4) REMARK: If $\mu^{\prime \prime}=\mu^{\prime}(\mathcal{L})$, then $I_{V}(\mathcal{L})=I_{W}(\mathcal{L}) \cap I_{\sigma}(\mathcal{L})$. This occurs if:

(a) $\mathcal{L}$ is countably compact (3.9),

(b) $\mu \in J(\mathcal{L})$ and $\mathcal{L}$ is $\delta(3.3 .4)$,

(c) $\mathcal{L}$ is normal and complement generated,

(d) $\mathcal{L}$ is $\delta$-normal.

(4.5) THEOREM: Suppose $\mathcal{L}$ is complement generated.

Then $J(\mathcal{L}) \subset I_{V}(\mathcal{L}) \subset I_{W}(\mathcal{L}) \cap I_{\sigma}(\mathcal{L})$. 
PROOF: Suppose $\mathcal{L}$ is complement generated and $\mu \in J(\mathcal{L})$; and let $L \in \mathcal{L}$ such that $\mu\left(L^{\prime}\right)=1$. Then $L=\bigcap_{n=1}^{x} L_{n}^{\prime}\left(L_{n} \in \mathcal{L}\right.$. all $\left.n\right), \mu \in I_{\sigma}(\mathcal{L})$, and $\mu^{\prime \prime}=\mu^{\prime}=\mu\left(\mathcal{L}^{\prime}\right)$ by $(3.10)$. This implies $L^{\prime}=\bigcup_{1}^{\infty} L_{n}$ and $\mu^{\prime \prime}\left(L^{\prime}\right)=1$. Also, $\mu^{\prime \prime}\left(L^{\prime}\right) \leq \sum_{n=1}^{\infty} \mu^{\prime \prime}\left(L_{n}\right)$ since $\mu^{\prime \prime}$ is an outer measure. Hence $\mu^{\prime \prime}\left(L_{n}\right)=1$ for some $n$. Thus $\mu \in I_{V}(\mathcal{L})$, which implies $\mu \in I_{W}(\mathcal{L})$ since $\mu^{\prime}\left(L_{n}\right)=1$ for some $n$. Therefore $J(\mathcal{L}) \subset I_{V}(\mathcal{L}) \subset I_{W}(\mathcal{L}) \cap I_{\sigma}(\mathcal{L})$.

(4.6) THEOREM: Suppose $\mathcal{L}$ is normal and $\mu \in M_{W^{\prime}}(\mathcal{L})$. Then $\mu \in M_{R}(\mathcal{L})$.

PROOF: Suppose $\mathcal{L}$ is normal and $\mu \in M_{W}(\mathcal{L})$. Let $\mu \leq \nu(\mathcal{L})$, where $\nu \in M_{R}(\mathcal{L})$. Then, using (3.11),

$$
\begin{aligned}
\nu\left(L^{\prime}\right) & =\sup \left\{\nu(\tilde{L}): \tilde{L} \subset L^{\prime} ; L, \tilde{L} \in \mathcal{L}\right\} \\
& =\sup \left\{\nu^{\prime}(\tilde{L}): \tilde{L} \subset L^{\prime} ; L, \tilde{L} \in \mathcal{L}\right\} \\
& =\sup \left\{\mu^{\prime}(\tilde{L}): \tilde{L} \subset L^{\prime} ; L, \tilde{L} \in \mathcal{L}\right\} \\
& =\mu\left(L^{\prime}\right) \text { since } \mu \in M_{W}(\mathcal{L}) .
\end{aligned}
$$

So $\mu=\nu\left(\mathcal{L}^{\prime}\right)$, which implies $\mu=\nu$ since $\mu(X)=\nu(X)$. Therefore $\mu \in M_{R}(\mathcal{L})$.

(4.7) COROLLARY: If $\mathcal{L}$ is normal and $\mu \in I_{W}(\mathcal{L})$, then $\mu \in I_{R}(\mathcal{L})$.

(4.8) THEOREM: Suppose $\mathcal{L}$ is normal and complement generated.

Then $\mu \in J(\mathcal{L})$ implies $\mu \in I_{R}(\mathcal{L})$.

PROOF: Suppose $\mathcal{L}$ is normal and complement generated; and let $\mu \in J(\mathcal{L})$. Then, by (4.5), $\mu \in I_{W}(\mathcal{L})$. Therefore $\mu \in I_{R}(\mathcal{L})$ by $(4.7)$.

(4.9) REMARK: We saw in Corollary(4.7) that if $\mathcal{L}$ is normal, then $I_{W}(\mathcal{L})=I_{R}(\mathcal{L})$. However, the converse is not true. For example, let $\mathcal{L}=\{\emptyset, X, A, B, A \cup B\}$, where $A, B \subset X(A, B \neq \emptyset)$ such that $A \cap B=\emptyset$ and $A \cup B \neq X$.

Here $\mathcal{L}$ is clearly not normal, but $I_{W}(\mathcal{L})=I_{R}(\mathcal{L})$.

(4.10) THEOREM: $(a) \mu \in I_{S}(\mathcal{L})$ if and only if $\mu=\mu^{\prime \prime}(\mathcal{L})$ and $\mu \in I_{\sigma}(\mathcal{L})$ (b) $\mu \in I_{S}(\mathcal{L})$ implies $\mu^{\prime \prime}=\mu^{\prime}=\mu\left(\mathcal{L}^{\prime}\right)$ and $\mu \in J(\mathcal{L})$

\section{PROOF:}

(a)

1.Suppose $\mu \in I_{S}(\mathcal{L})$. Then $\mu \in I_{\sigma}(\mathcal{L})$, by definition, and hence $\mu \leq \mu^{\prime \prime}(\mathcal{L})$. Now let $\mu(L)=0$, where $L \in \mathcal{L}$. Then $\mu\left(L^{\prime}\right)=1$. Since $\mu \in I_{S}(\mathcal{L}), L^{\prime} \supset \bigcap_{n=1}^{\infty} L_{n}$ and $\mu\left(L_{n}\right)=1$, all $n, L_{n} \in \mathcal{L}$. In other words $L \subset \bigcup_{1}^{\infty} L_{n}^{\prime}$ and $\mu\left(L_{n}^{\prime}\right)=0$, all $n$. Hence $\mu^{\prime \prime}(L)=\inf \left\{\sum_{n=1}^{\infty} \mu\left(L_{n}^{\prime}\right): L \subset \cup L_{n}^{\prime}, L_{n} \in \mathcal{L}\right\}=0$. Therefore $\mu=\mu^{\prime \prime}(\mathcal{L})$.

2. Suppose $\mu \in I_{\sigma}(\mathcal{L})$ and $\mu=\mu^{\prime \prime}(\mathcal{L})$. Let $\mu\left(L^{\prime}\right)=1, L \in \mathcal{L}$. Then $\mu(L)=0$, which implies $\mu^{\prime \prime}(L)=0$. Thus there exists $L_{n} \in \mathcal{L}, n=1,2, \ldots$, such that $L \subset \cup L_{n}^{\prime}$ and $\mu\left(L_{n}^{\prime}\right)=0$, all $n$. Hence $\mu\left(L_{n}\right)=1$, all $n$; and $L \subset \bigcup_{n=1}^{\infty} L_{n}^{\prime}$ implies $L^{\prime} \supset \bigcap_{n=1}^{\infty} L_{n}$. Therefore $\mu \in I_{S}(\mathcal{L})$. 
(b) Suppose $\mu \in I_{S}(\mathcal{L})$. Then $\mu \in I_{\sigma}(\mathcal{L})$, and hence $\mu^{\prime \prime} \leq \mu=\mu^{\prime}\left(\mathcal{L}^{\prime}\right)$. Now suppose $\mu^{\prime \prime}\left(L^{\prime}\right)=0$ and $\mu^{\prime}\left(L^{\prime}\right)=1$. Then $L^{\prime} \supset \bigcap_{n=1}^{\infty} L_{n}$ such that $\mu\left(L_{n}\right)=1$, all $n, L_{n} \in \mathcal{L}$. In other words $L \subset \bigcup_{1}^{\infty} L_{n}^{\prime}$ such that $\mu\left(L_{n}^{\prime}\right)=0$, all $n$. Consequently $\mu^{\prime \prime}(L)=0$. Thus $1=\mu^{\prime \prime}(X) \leq \mu^{\prime \prime}(L)+\mu^{\prime \prime}\left(L^{\prime}\right)=0$, a contradiction. Therefore $\mu^{\prime \prime}=\mu^{\prime}=\mu\left(\mathcal{L}^{\prime}\right)$, and $\mu \in J(\mathcal{L})$ by $(3.10)$.

(4.11) THEOREM: If $\mu \in I_{S}(\mathcal{L})$, then $\sigma(\mathcal{L}) \subset \mathcal{S}_{\mu^{\prime \prime}}$ (where $\sigma(\mathcal{L})$ is the $\sigma$-algebra generated by $\mathcal{L}$ ).

PROOF: Suppose $\mu \in I_{S}(\mathcal{L})$, which implies $\mu \in I_{\sigma}(\mathcal{L})$, and let $L \in \mathcal{L}$. Now if $\mu(L)=0$, then $\mu^{\prime \prime}(L)=0$, which implies $L \in \mathcal{S}_{\mu^{\prime \prime}}$. If instead $\mu(L)=1$, then $\mu\left(L^{\prime}\right)=0$, which implies $\mu^{\prime \prime}\left(L^{\prime}\right)=0$ by $(4.10(b))$. Thus $L^{\prime} \in S_{\mu^{\prime \prime}}$, and once again $L \in \mathcal{S}_{\mu^{\prime \prime}}$. Therefore $\sigma(\mathcal{L}) \subset \mathcal{S}_{\mu^{\prime \prime}}$ since $\mathcal{S}_{\mu^{\prime \prime}}$ is a $\sigma$-algebra.

\section{(4.12) LEMMA: $I_{R}^{\sigma}(\mathcal{L}) \subset I_{S}(\mathcal{L})$}

(4.13) THEOREM: If $\mu \in I_{S}(\mathcal{L})$ and $\mathcal{L}$ is $\delta$, then $\mu \in I_{R}^{\sigma}(\mathcal{L})$.

PROOF: Suppose $\mu \in I_{S}(\mathcal{L})$ and $\mathcal{L}$ is $\delta$. Let $\mu\left(L^{\prime}\right)=1, L \in \mathcal{L}$. Then there exists $L_{n} \in \mathcal{L}$ such that $L^{\prime} \supset \bigcap_{1}^{\infty} L_{n}=\tilde{L} \in \mathcal{L}$ and $\mu\left(L_{n}\right)=1$, all $n$. Hence $\mu\left(L_{n}^{\prime}\right)=0$, all $n$. We know $\mu \in I_{S}(\mathcal{L})$ implies $\mu \in I_{\sigma}(\mathcal{L})$ and $\mu^{\prime \prime}=\mu=\mu^{\prime}\left(\mathcal{L}^{\prime}\right)$. Consequently $\mu^{\prime \prime}\left(L_{n}^{\prime}\right)=0$, all $n$, but $\cup L_{n}^{\prime} \uparrow \tilde{L}^{\prime}$. Hence $\mu^{\prime \prime}\left(\tilde{L}^{\prime}\right)=0$ since $\mu^{\prime \prime}$ is a regular outer measure. This implies $\mu(\tilde{L})=1$. Thus $\mu \in I_{R}(\mathcal{L})$. Therefore $\mu \in I_{R}^{\sigma}(\mathcal{L})$.

(4.14) REMARK: We see in Lemma(4.12) and Theorem(4.13) that if $\mathcal{L}$ is $\delta$, then $I_{S}(\mathcal{L})$ $=I_{R}^{\sigma}(\mathcal{L})$. Therefore, by $\operatorname{Lemma}(4.3), I_{S}(\mathcal{L}) \subset I_{V}(\mathcal{L}) \subset I_{W}(\mathcal{L}) \cap I_{\sigma}(\mathcal{L})$, in this case.

(4.15) THEOREM: Suppose $\mathcal{L}_{1}$ and $\mathcal{L}_{2}$ are two lattices of subsets of $X$ such that $\mathcal{L}_{1} \subset \mathcal{L}_{2}$. Let $\nu \in I_{W}\left(\mathcal{L}_{2}\right)$. If $\mathcal{L}_{1}$ semi-separates $\mathcal{L}_{2}$, then $\mu \in I_{W}\left(\mathcal{L}_{1}\right)$ (where $\left.\mu=\left.\nu\right|_{A\left(\mathcal{L}_{1}\right)}\right)$.

PROOF: Given $\mathcal{L}_{1} \subset \mathcal{L}_{2}$ such that $\mathcal{L}_{1}$ semi-separates $\mathcal{L}_{2}$ and $\nu \in I_{W}\left(\mathcal{L}_{2}\right)$. Then $\mu=$ $\nu \mid \in I\left(\mathcal{L}_{1}\right)$. Now suppose $\mu\left(L_{1}^{\prime}\right)=1, L_{1} \in \mathcal{L}_{1}$. Then $\nu\left(L_{1}^{\prime}\right)=1$. Since $\nu \in I_{W}\left(\mathcal{L}_{2}\right)$ there exists $L_{2} \in \mathcal{L}_{2}$ such that $L_{1}^{\prime} \supset L_{2}$ and $\nu^{\prime}\left(L_{2}\right)=1$. By semi-separation there exists $\tilde{L}_{1} \in \mathcal{L}_{1}$ such that $\tilde{L}_{1} \supset L_{2}$ and $L_{1} \cap \tilde{L}_{1}=\emptyset$. Hence $\nu^{\prime}\left(\tilde{L}_{1}\right)=1$, which implies $\mu^{\prime}\left(\tilde{L}_{1}\right)=1$ since $\nu^{\prime} \leq \mu^{\prime}(\mathcal{L})$. Therefore $\mu \in I_{W}\left(\mathcal{L}_{1}\right)$.

(4.16) THEOREM: Suppose $\mathcal{L}_{1}$ and $\mathcal{L}_{2}$ are two lattices of subsets of $X$ where $\mathcal{L}_{1} \subset \mathcal{L}_{2}$. Let $\nu \in I_{V}\left(\mathcal{L}_{2}\right)$. If $\mathcal{L}_{1}$ semi-separates $\mathcal{L}_{2}$, then $\mu \in I_{V}\left(\mathcal{L}_{1}\right)\left(\right.$ where $\left.\mu=\left.\nu\right|_{A\left(\mathcal{L}_{1}\right)}\right)$.

PROOF: The proof is similar to that of Theorem(4.15) and will be omitted.

(4.17) THEOREM: Suppose $\delta\left(\mathcal{L}^{\prime}\right)$ separates $\mathcal{L}$.

Then $\mu \in I_{\sigma}\left(\mathcal{L}^{\prime}\right) \cap I_{W}(\mathcal{L})$ implies $\mu \in I_{R}(\mathcal{L})$.

PROOF: Suppose $\delta\left(\mathcal{L}^{\prime}\right)$ separates $\mathcal{L}$. Let $\mu \in I_{\sigma}\left(\mathcal{L}^{\prime}\right) \cap I_{W}(\mathcal{L})$ such that $\mu \leq \nu(\mathcal{L})$, where $\nu \in I_{R}(\mathcal{L})$. Now suppose $\mu \neq \nu$. Then there exists $A \in \mathcal{L}$ such that $\nu(A)=1$ and 
$\mu(A)=0$. which implies $\mu\left(A^{\prime}\right)=1$. Thus $A^{\prime} \supset B \in \mathcal{L}$ where $\mu^{\prime}(B)=1$ since $\mu \in I_{W}(\mathcal{L})$. Now $\delta\left(\mathcal{L}^{\prime}\right)$ separates $\mathcal{L}$ inplies:

$$
\begin{aligned}
& \text { (1) } A \subset \bigcap_{n=1}^{\infty} L_{n}^{\prime}, L_{n} \in \mathcal{L} \text {, all } n \\
& \text { (2) } B \subset \bigcap_{m=1}^{\infty} \tilde{L}_{m}^{\prime}, \tilde{L}_{m} \in \mathcal{L} \text {, all } m \\
& \text { (3) } \cap \bigcap_{n, m}^{\prime \prime} L_{n}^{\prime} \cap \tilde{L}_{m}^{\prime}=\emptyset
\end{aligned}
$$

It follows that $\nu(A)=1$ implies $\nu\left(L_{n}^{\prime}\right)=1$, all $n$, which implies $\mu\left(L_{n}^{\prime}\right)=1$, all $n$. Also $\mu^{\prime}(B)=1$ implies $\mu\left(\tilde{L}_{m}^{\prime}\right)=1$, all $m$. Hence $\mu\left(L_{n}^{\prime} \cap \tilde{L}_{m}^{\prime}\right)=1$, all $n, m$; and recall $\bigcap_{n, m} L_{n}^{\prime} \cap \tilde{L}_{m}^{\prime}=\emptyset$. This is a contradiction since $\mu \in I_{\sigma}\left(\mathcal{L}^{\prime}\right)$. Thus $\mu=\nu$. Therefore $\mu \in I_{R}(\mathcal{L})$.

(4.18) THEOREM: $\mu \in I_{S}(\mathcal{L})$ if and only if there exists $\nu \in I_{R}[\delta(\mathcal{L})]$ such that $\left.\nu\right|_{A(\mathcal{L})}=\mu$.

\section{PROOF:}

1. Suppose $\mu \in I_{S}(\mathcal{L})$. Then $\mu \in I_{\sigma}(\mathcal{L}), \mu^{\prime \prime}=\mu(\mathcal{L})$, and $\mathcal{S}_{\mu^{\prime \prime}} \supset \sigma(\mathcal{L})$ (which implies $\left.\mathcal{S}_{\mu^{\prime \prime}} \supset A[\delta(\mathcal{L})]\right)$. Let $\bar{\mu}=\left.\mu^{\prime \prime}\right|_{\boldsymbol{S}^{\prime \prime}}$. Then $\bar{\mu}$ is countably additive and $\left.\bar{\mu}\right|_{A[\delta(\mathcal{L})]}=\nu \in$ $I_{R}[\delta(\mathcal{L})]$. Now suppose $\nu\left(D^{\prime}\right)=1, D \in \delta(\mathcal{L})$. Then, since $D \in \delta(\mathcal{L}), D=\bigcap_{n=1}^{\infty} L_{n}, L_{n} \in \mathcal{L}$. It follows that $1=\nu\left[\left(\bigcap_{n=1}^{\infty} L_{n}\right)^{\prime}\right]=\nu\left(\cup L_{n}^{\prime}\right)=\mu^{\prime \prime}\left(\cup L_{n}^{\prime}\right) \leq \sum \mu^{\prime \prime}\left(L_{n}^{\prime}\right)$. Hence $\mu^{\prime \prime}\left(L_{N}^{\prime}\right)=1$, some $N$, which implies $\nu\left(L_{N}^{\prime}\right)=1$ and $\mu\left(L_{N}^{\prime}\right)=1$, some $N$. Also $\left(\cap L_{n}\right)^{\prime}=\cup L_{n}^{\prime} \supset L_{N}^{\prime} \supset$ $\cap A_{n}, A_{n} \in \mathcal{L}$; and $\mu\left(A_{n}\right)=1$, all $n$, since $\mu \in I_{S}(\mathcal{L})$. Thus $\nu\left(\cap A_{n}\right)=\mu^{\prime \prime}\left(\cap A_{n}\right)=1$ and $\cap A_{n} \in \delta(\mathcal{L})$. Therefore $\nu \in I_{R}[\delta(\mathcal{L})]$.

2. The proof of the converse will be omitted.

\section{REFERENCES}

[1A] A. D. ALEXANDROFF (ALEKSANDROV), Additive set-functions in abstract spaces (Chapter I), Mat. Sb. 8 (1940), 307-348. MR 2-315.

[1B] A. D. ALEXANDROFF (ALEKSANDROV), Additive set-functions in abstract spaces (Chapters II-III), Mat. Sb. 9 (1941), 563-628. MR 3-207.

[1C] A. D. ALEXANDROFF (ALEKSANDROV), Additive set-functions in abstract spaces (Chapters IV-VI), Mat. Sb. 13 (1943), 169-238. MR 6-275.

[2] J. Camacho, Jr., On maximal measures with respect to a lattice, Internat. Jour. Math. G Math. Sci. 13 no.1 (1991), 93-98.

[3] G. Eid, On maximal measures and Wallman spaces, Internat. Jour. Math. 63 Math. Sci. 13 no.1 (1990), 31-38.

[4] Z. Frolik, Prime filters with the C.I.P., Comm. Math. Univ. Carolinae 13 (1972), 553-575.

[5] M. SZETO, Measure repleteness and mapping preservations, Jour. of the Indian Math. Soc. 43 (1979), 35-52.

[6] M. SZETO, On separation of lattices, Internat. Jour. Math. E Math. Sci. 14 no. 2 (1991), 325-338. 


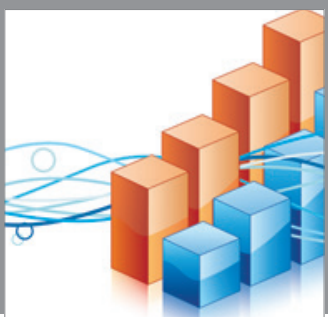

Advances in

Operations Research

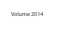

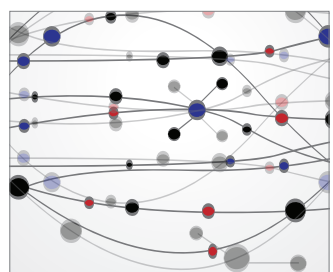

\section{The Scientific} World Journal
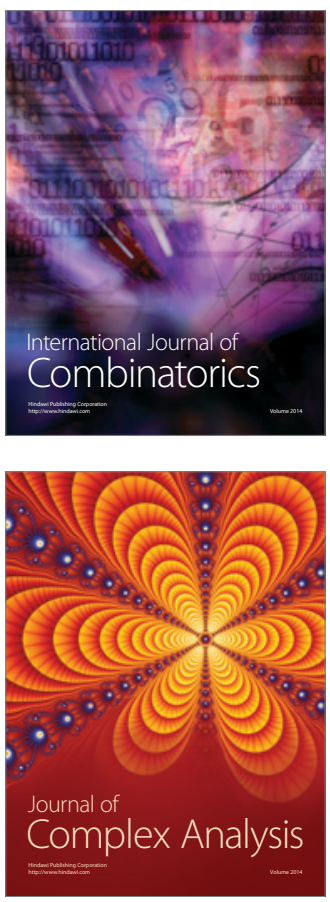

International Journal of

Mathematics and

Mathematical

Sciences
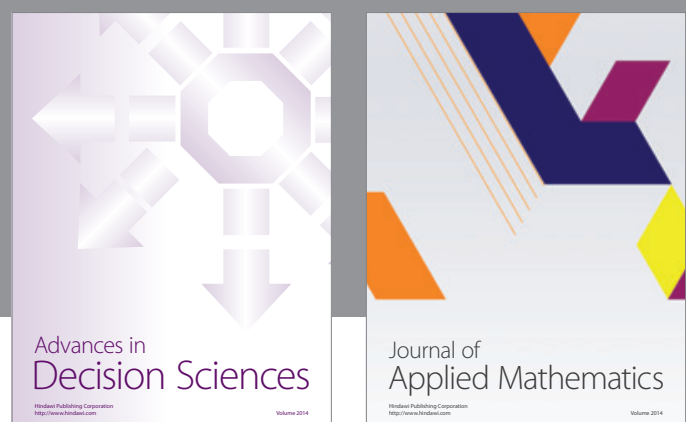

Journal of

Applied Mathematics
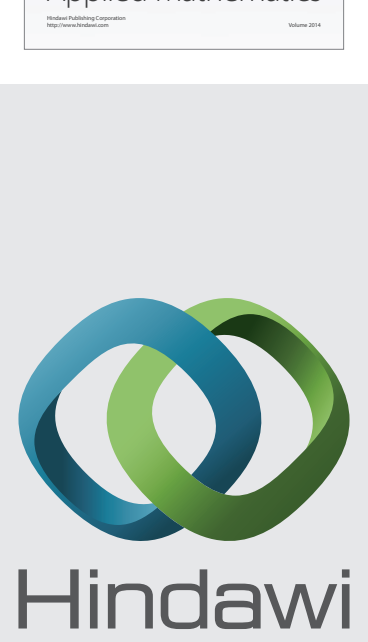

Submit your manuscripts at http://www.hindawi.com
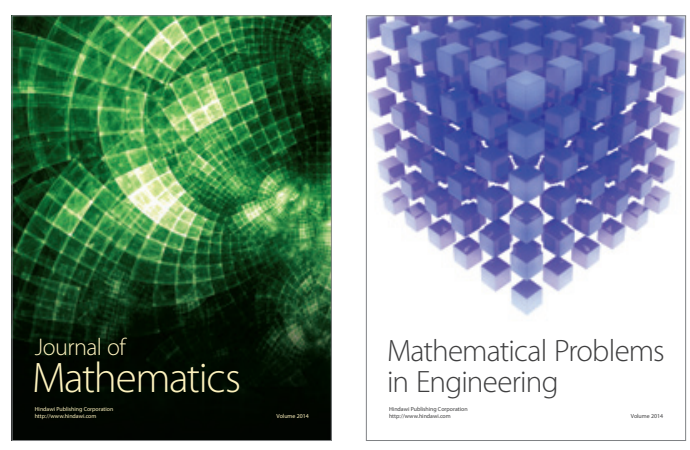

Mathematical Problems in Engineering
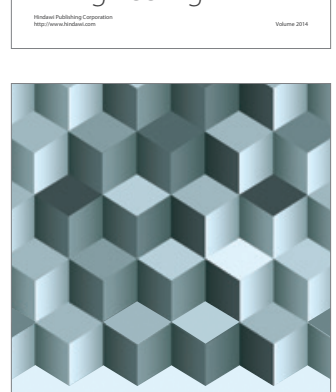

Journal of

Function Spaces
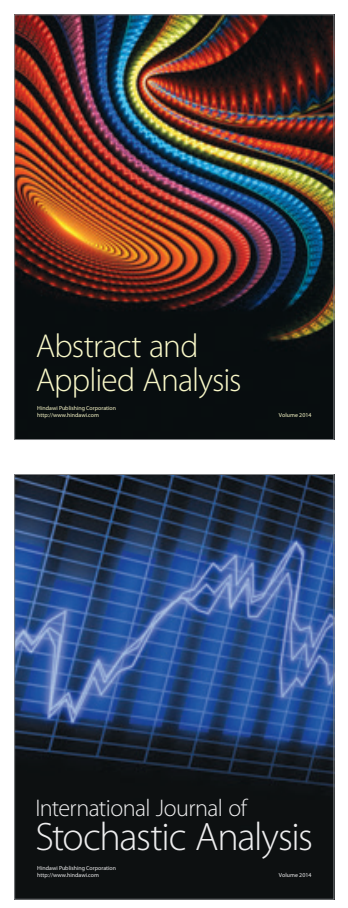

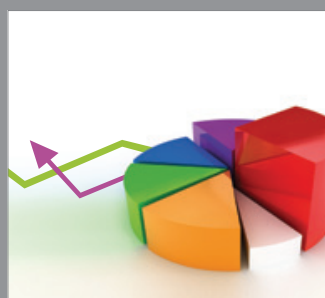

ournal of

Probability and Statistics

Promensencen
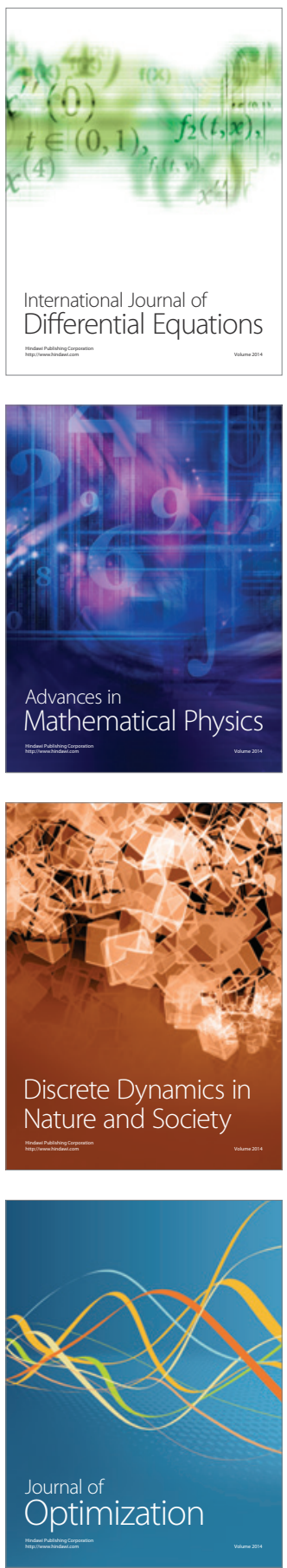